\title{
What is the difference between a spinning neutron star and a spinning 'black hole'? - or - evidence for a 1:1 resonance coupling between the rotation/spin frequency and the radial epicyclic frequency at extreme Kerr values of angular momentum.
}

\section{Bernd Aschenbach ${ }^{1}$}

$P R$ Vaterstetten

Mozartstrasse 8, 85591 Vaterstetten, Germany

E-mail: bernd.aschenbachet-online.de

Two distinct relations between QPO frequency $\boldsymbol{f}$ (Low \& High frequencies) and 'black hole' mass $\mathbf{M}$ have been established by observation (Smith et al., 2018). M· $\boldsymbol{f}=$ const with different constants for the $\boldsymbol{L}$ and $\boldsymbol{H}$ relation. The 'black hole' attributed QPOs agree at high frequencies with the spin frequency of the most rapidly rotating neutron stars of the two classes of 'the ordinary' pulsars and the ms-pulsars. 'Ordinary' pulsars fit the $\boldsymbol{L}-\boldsymbol{f}$ relation and the ms-pulsars follow the $\boldsymbol{H}$ - $\boldsymbol{f}$ relation, permitting some calculation of their neutron star mass. This is illustrated for the Crab pulsar ('ordinary') and the ms-pulsar J1748-244ad. This observation which suggests a physical link between the spin frequency of extremely rotating neutron stars and QPO frequencies of 'black holes' is likely to be a consequence of the 'Aschenbach' effect, being effective at Kerr parameters of $a>0.9953$ (Aschenbach, 2004).

Multifrequency Behaviour of High Energy Cosmic Sources - XIII - MULTIF2019

3-8 June 2019

Palermo, Italy

\footnotetext{
${ }^{1}$ Speaker
} 


\section{Introduction}

Generally speaking, the frequency of the appearance of pulsed emission from a pulsar is attributed to the rotation of a neutron star such that the period of the pulses' appearance is identical to the rotation period of the neutron star and the site of emission is located at some place in the magnetosphere of the neutron star. The spin periods or spin frequencies $v_{S}$ of pulsars come in two distinct sets (e.g., Becker, Kramer \& Sesana, 2018), which differ significantly in $v_{s}$ grouping the pulsars in so called 'ordinary' pulsars, like the Crab pulsar with a spin period of 33 ms or $v_{s}=30 \mathrm{~Hz}$, and the 'millisecond' pulsars, the fastest spinning of which, J1748-244ad, has a period of $1.4 \mathrm{~ms}$ or $v_{S}=716 \mathrm{~Hz}$.

'Black holes' have been found to exhibit quasiperiodic oscillations (QPOs). For a subset of 'black holes' a tight correlation between the 'black hole' mass $M$ and the frequency $f_{q}$ of the QPOs have been found such that the product $M \cdot f_{q}=$ const across 7 orders of magnitude concerning $M$ and $f_{q}$ alike. These QPOs come in two distinct sets, which differ in $f_{q}$ by about a factor of $\approx 40$ for the same $M$. Accordingly, they are named high frequency QPOs (HFQPO) and low frequency QPOs (LFQPO). Notably, both the LFQPOs and the HFQPOs relations $M \cdot f_{q}=$ const have been observed holding over at least 7 orders of magnitude. Best fits to the observed correlations are $M \cdot f_{q}=1902 \mathrm{M}_{\odot} / \mathrm{s}$ for the HFQPOs and $M \cdot f_{q}=52 \mathrm{M}_{\odot} / \mathrm{s}$ for the LFQPOs (Smith et al. 2018).

I want to point out that the application of the $M \cdot f_{q}=$ const QPO relations observed for 'black hole' sources down to the neutron mass regime shows that $f_{q} \sim v_{s}$ for the fastest spinning pulsars. The LFQPO $f_{q}$ is associated with the spin frequency $v_{s}$ of the fastest spinning 'ordinary' pulsar and the HFQPO $f_{q}$ is associated with the spin frequency $v_{s}$ of the fastest spinning millisecond pulsar. Though despite the tight similarity of the values for $f_{q}$ and $v_{s}$ I note that there is a significant difference between pulsar periods and 'black hole' QPOs apparent in the power density spectra (PDS) as the PDSs of the neutron stars are being claimed of showing a delta function like peak whereas the 'black hole' PDSs frequency excess is broadened, although a clear distinction is missing because of lack of a definition of a QPO signature. The respective PDSs need to be analysed in detail and physically understood, but see Aschenbach, 2018, for a suggestion.

The $M \cdot f_{q}=$ const relations for both the LFQPOs and the HFQPOs of 'black holes' have been modeled. Multiple resonances between the three spherical coordinate/epicyclic oscillations using the Kerr metric have been found and their eigenfrequencies have been determined (Aschenbach, $2004 \&$ 2018). The model predictions match the observation based best fits by better than a few percent and encourage an interpretation along the epicyclic resonance model.

In the following sections the QPO resonance model is revisited and the results are presented which, among other notable findings like the involvement of the Planck mass, Planck time and the number $\pi$, explain $f_{q}=v_{s}$ by $f_{q}$ being due to the radial coordinate oscillation mode, the classical radial epicyclic oscillation, by which oscillation in the orbital/equatorial plane of the rotating object is described. 


\section{The QPO epicyclic resonance model and its application to neutron stars}

The model for the $M \cdot f_{q}=$ const QPO relations employs the three spherical coordinate oscillations. The oscillation modes comprise a rotation around some axis measured by an angle $\varphi$, by which the equatorial plane is set, and the two epicyclic oscillation modes, associated with the radial coordinate $r$ and the angular polar coordinate $\theta$. They describe oscillations in the equatorial plane $(r)$ and perpendicular to the orbital plane $(\theta)$. The dimensionless frequencies $\Omega_{\varphi}, \Omega_{\theta}$ and $\Omega_{r}$ depend, in the Kerr metric, on just $r$ and $a$, the latter of which is the measure of angular momentum. The relevant equations can, e.g., be found in the paper of Aschenbach (2004).

A resonance between any two of these three modes marks a stable or, for some time, quasistable state. Therefore a resonance between some two of the modes has been considered for providing deeper understanding of the impact of Strong Gravity on 'black hole' physics. For a brief review and the numerous references and authors involved see Aschenbach (2004 \& 2018).

A single resonance between just two oscillations does not constrain or eventually fix the involved parameters, but the occurrence of multiple resonances would do. Scanning the Kerr parameter $a$, just two cases for multiple resonances among the coordinate frequencies have been found. The results of the scans have been reported in Aschenbach, 2004, and Aschenbach, 2018. Such multiple resonance conditions are given for $a l=0.99623$ and for $a h=0.99616$.

For $a=a h$ the resonance conditions are $\left\{\Omega_{\theta} / \Omega_{r}=3: 1\right.$ at a radius $r=r_{31}, \Omega_{\theta} / \Omega_{r}=3: 2$ at $r=r_{32}$ and $\left.\Omega_{\varphi}\left(r_{31}\right) / \Omega_{\varphi}\left(r_{32}\right)=3: 1\right\}$. The latter resonance between $\Omega_{\varphi}\left(r_{31}\right)$ and $\Omega_{\varphi}\left(r_{32}\right)$ implies, in the classical astronomical context, the existence of two commensurable orbits. These three resonance conditions do not only explain the 3:2 twin peak QPOs but they provide a quantitative prediction for the HFQPOs $M \cdot f_{q}=$ const QPO relation, which differs from the best fit to the observations by a few percent.. For the lower 3:2 twin peak frequency the QPO is due to the radial mode oscillation and for the higher frequency it is the polar mode oscillation, occurring at the radius $r_{32}$.

For $a=a_{l}$ the resonance conditions are $\left\{\Omega_{\varphi} / \Omega_{\theta}=3: 1\right.$ and $\Omega \varphi / \Omega_{r}=13: 1$ at $r=r_{131}$ and $\left.\Omega_{r}\left(r_{131}\right) / \Omega_{r}\left(r_{0}\right)=18: 1\right\}$. At the time of writing I have not yet found a physical explanation for the 18:1 resonance other than an argument concerning the number 18 being a natural number, which is composed of just the numbers 2 and 3. These low natural numbers are essential in resonance/interference/coherence phenomena of oscillations and waves. Regardless of that, this latter resonance condition provides a almost perfect match of the model to the data for the LFQPOs $M \cdot f_{q}=$ const relation. The value of the resonance radius $r_{0}$ is marginally above the innermost stable circular orbit (ISCO) for $a=a_{l}$.

The values of the $r^{\prime}$ s and $\Omega$ 's as such have no physical scale attached, they have been derived using $\mathrm{c}=\mathrm{G}=1$. The physical length scales are in units of $\mathrm{G} M / \mathrm{c}^{2}$, such that $\mathrm{r}=1$ is the gravitational radius $r_{\mathrm{g}}$. Angular frequencies $\Omega$ are in units of $\mathrm{c}^{3} /(\mathrm{G} M)$. The conversion of $\Omega$ to an observable frequency $f$ is $f=\Omega \mathrm{c}^{3} /(2 \pi \mathrm{G} M)$.

The numerical values of the $r^{\prime}$ s and $\Omega^{\prime}$ s effective in the resonances are provided for the HFQPO's in Aschenbach 2004, and for the LFQPO's in Aschenbach 2018. These relations 
between mass and frequency or mass and period observed both for LFQPOs and HFQPOs, and a possible physical implication are discussed in the following sections. The relations appear to be not constrained in mass and have been observed for 'black holes' over more than 7 orders in magnitude of mass from stellar type mass to supermassive objects. But 'black holes' are expected to come at any mass, down to primordial, low mass 'black holes' up to the most supermassive 'black holes'. Timing studies of extremely supermassive objects in terms of QPOs are difficult because of the expected extremely long periods, although supermassive objects like clusters of galaxies are known to be associated with dark matter. Timing studies of the mass-frequency relation towards lower mass range, i.e., the mass of neutron stars, however, are possible and this may provide some more information.

\subsection{The Crab pulsar and the LFQPO relation}

The model for the LFQPO mass-frequency relation of the radial or r-mode epicyclic oscillation reads $M \cdot f_{r}=51.9 \mathrm{M}_{\odot} / \mathrm{s}$. Because of the extreme value of the angular momentum involved ( $a=0.99623)$ the spin-down rate of the neutron star needs to be taken into account before applying the suspected mass-frequency relation to get as near as possible to the nascent state of the pulsar at its birth. The nascent spin period of the neutron star can then be tried to be estimated by its current spin period of $33 \mathrm{~ms}$ and the spin-down rate of $38 \mathrm{~ns} / \mathrm{day}$, which gives a nascent spin period of $19.6 \mathrm{~ms}$ for an age of 965 years. Applying the mass-frequency relation the mass of a 'back hole' like neutron star for the Crab pulsar would be $1 \mathrm{M}_{\odot}$. Neglecting the spin-down rate because of the unknown glitch history and other unknown spin-down mechanisms in the very early times after birth an upper limit of the mass of $1.7 \mathrm{M}_{\odot}$ is obtained. This would agree with the assumption $f_{q}=f_{r}=v_{s}$.

If rotating neutron stars follow the Kerr metric they should have an innermost marginally stable orbit (ISCO). This is independent of any equation of state employed for the matter. For a neutron star of $1 \mathrm{M}_{\odot}$ with $a=0.99623 r_{\text {ISCO }}=1.9265 \mathrm{~km}$. And as for a 'black hole' of $1 \mathrm{M}_{\odot}$ with $a=0.99623$ there is an unexplained, unexplored territory of mass repective energy. If this core below the ISCO consisted of 'quarks' or any other type of matter in the case of a neutron star for $r<r_{\text {ISCO }}$ I would expect this type of matter to also populate the region below the ISCO of 'black holes' - or, there is no rest-mass matter but energy below $r_{\text {ISCO }}$. In case of an object of $1 \mathrm{M}_{\odot}$ with $a=0.99623$ the r-mode resonance is localized at a radius above the ISCO by $0.5 \mathrm{~m}$.

\subsection{The hitherto fastest millisecond pulsar and the HFQPO relation}

The model for the HFQPO mass-frequency relation of the radial or r-mode epicyclic oscillation for $a_{h}=0.99616$ reads $M \cdot f r=2030.8 \mathrm{M}_{\odot} / \mathrm{s}$. As mentioned in section 2.1 the relation is meant to provide an upper limit for the spin frequency $v_{s}$. The HFQPO relation is suggested to apply to the millsecond pulsars. The fastest ms-pulsar known at the time of writing is PSR J1748-244ad (Hessels et al., 2006) with $v_{s}=716 \mathrm{~Hz}$. This pulsar is member of an eclipsing binary system and the mass is not determined, but according to Hessels et al. the radius $\mathrm{R}$ of the neutron star needs to be less than $16 \mathrm{~km}$ for an assumed mass of $2 \mathrm{M}_{\odot}$. Otherwise it will start loosing mass from its surface assuming the maximum spin relation of Lattimer and Prakash 
(2004). Applying the HFQPO relation with $f_{q}=f_{r}=v_{s}=716 \mathrm{~Hz}$ a 'black hole' like object would have a mass of $2.8 \mathrm{M} \odot$, unless the HFQPO relation would be cut at its low mass end. The mass of $2.8 \mathrm{M} \odot$ is fairly close to what is expected to be the upper mass limit for a neutron star. It looks as if a discrimination between the maximum neutron star state and the 'black hole' state is possible by looking for a change in a ms-pulsar period pattern towards the HFQPO 'black hole' PDS. In addition to the mass involved radii are calculated. For $2.8 \mathrm{M} \odot$ the radius $\mathrm{R}$ of the neutron star needs to be $\mathrm{R}<18.1 \mathrm{~km}$ according to the Lattimer \& Prakash mass-shedding relation. For the 'black hole' model the r-mode resonance would have to arise at a lower radius to become effective. The resonance model result is $r=16.2 \mathrm{~km}$. There is no conflict between the neutron star state and the so-called black hole' state. A neutron star is as compact as a 'black hole' as far as mass and radius are concerned, for $a->1$. I think this invalidates the absolutism of the 'no-hair' theorem associated with 'black holes'. There is more than just mass and radius. This is likely to be magnetic energy, not electric charge, as the neutron is involved, which has no electric charge. And there are active r-mode oscillations which are likely to initiate waves propagating through the 'black hole' matter above the ISCO (Aschenbach, 2018).

\section{Interpretation of the constant of the QPO mass- frequency relation}

Observations have shown that there exists a fairly strict relation for 'black holes' between the QPO frequency and the 'black hole' mass. Looking at QPO frequencies expected in the range of neutron star masses it is found that the r-mode QPO frequencies are very similar if not identical with the rotational spin frequencies of the neutron star pulsars both for the most rapidly spinning 'ordinary' pulsar, the Crab pulsar, and for the most rapidly spinning ms-pulsar, PSR J1748.2546ad. There is only a fairly limited number of 'black holes' following the massfrequency relation and it is only the most fast rotating pulsars which match the relation. This suggests a selection effect.

The model of resonance modes of the coordinate (epicyclic) oscillations (Aschenbach, $2004 \& 2018$ ) match the observations to better than a few percent. According to this model the selection is because of the extremely close-to-one value of the Kerr angular momentum $a$. Pulsars with lower than the maximal spin are not affected, they are likely to have lower $a^{\prime}$ s, and they will not follow the mass-frequency relation, likewise do the lower spin 'black holes'. So the vast majority of neutron star pulsars and 'black holes' are not adressed and remain a sort of continuum population not affected by the resonances.

The dimensionless frequencies found for the 3:2 and 3:1 HFQPO resonances between the $r$-mode and the $\theta$-mode are listed in Table 1 of my paper of 2004 (Aschenbach, 2004). They have been obtained by numerical calculations. A closer look at the values and a more precise calculation show that the values for the $\Omega$ 's are consistent with multiples of the number $\pi:\left\{\Omega_{r}\right.$ $=[2 \pi / 100], \Omega_{\theta}=3 / 2[2 \pi / 100]$ for the $3: 2$ resonance $\}$ and $\left\{\Omega_{r}=3 / 4[2 \pi / 100]=3 / 2^{2}[2 \pi / 100]\right.$, $\Omega_{\theta}=9 / 2[2 \pi / 100]=\left(3^{2} / 2\right)[2 \pi / 100]$ for the $3: 1$ resonance $\}$. I note that the factors in front of $[2 \pi / 100]$ are built by just the natural numbers 2 and 3 , completed by the natural number $100=$ $\left(5^{*} 2\right)^{2}$. The conversion from dimensionless frequencies $\Omega$ to observable frequencies $f$ is $f=\Omega$ / $(2 \pi) \mathrm{x} \mathrm{c}^{3} /(\mathrm{GM})$, such that the factor $(2 \pi)$ cancels when observables instead of dimensionless 
quantities are used. Only the natural numbers 2, 3, 5 and, possibly, the logarithm operator remain. So the mass-frequency relation for $a=a_{h}$ can be expressed by a set of natural numbers.

The relation $M \cdot f_{q}=$ const for the radial epicylic oscillation mode at $r=r_{32}$ then reads $M \cdot f_{r}=(1 / 100) \cdot\left(\mathrm{c}^{3} / \mathrm{G}\right)$. The term $\mathrm{c}^{3} / \mathrm{G}$, which remains explaining, is per definition $\mathrm{c}^{3} / \mathrm{G}=\mathrm{m}_{\mathrm{P}} / \mathrm{t}_{\mathrm{P}}$ with $m_{P}$ the Planck mass and $t_{P}$ the Planck time, which are defined as $m_{P}=\sqrt{ }(\hbar c / G)$ and $t_{P}=$ $\sqrt{ }\left(\hbar \mathrm{G} / \mathrm{c}^{5}\right)$, employing just the three fundamental constants $\mathrm{c}, \mathrm{G}$ and $\hbar$. This means that, for instance, the 3:2 radial r-mode resonance frequency $f_{r}=(1 / 100) \mathrm{m}_{\mathrm{P}} / \mathrm{t}_{\mathrm{P}}$. In this context $\mathrm{t}_{\mathrm{P}}$ is meant to be a periodic time scale. This can be written, with $P_{r}=1 / f_{r^{\prime}}$ and $P_{r}$ a periodic time scale, as $\left\{P_{r} / \mathrm{t}_{\mathrm{p}}\right\} /\left\{M / \mathrm{m}_{\mathrm{P}}\right\}=100$. The ratio of the period of the radial epicyclic or radial coordinate oscillation mode measured in units of the Planck time divided by the mass $M$ measured in units of the Planck mass is equal to the natural number 100, whereby $M$ stands for mass in the Kerr metric, not necessarily the mass of a 'black hole'.

The involvement of low natural numbers and the Planck scales may be a grand coincidence with no physical background, at all. But it may point to a gravity quantum effect imposed by the extreme values of angular momentum in terms of the Kerr parameter and the extreme compactness expressed by the ratio $\mathrm{M} / \mathrm{R}$.

I would like to point out that the values for the Kerr angular momenta $a=a_{l}$ and $a=a_{h}$, which determine the resonance freqencies, are larger than the critical value of $a=a_{c}=0.9953$ (Aschenbach, 2004). At the value of $a_{c}$ the Kepler relation between orbital velocity $\mathrm{v}_{\varphi}$ and radius $r$ changes dramatically. Whereas for $a<a_{c}$ the Kepler law applies in the sense that $\mathrm{v}_{\varphi}$ deceases with increasing radius the opposite happens for $a>a_{\mathrm{c}}$. The orbital velocity $\mathrm{v}_{\varphi}$ decreases with deceasing radius (Aschenbach, 2004). Furthermore the $\mathrm{v}_{\varphi}{ }^{-r}$ relation shows a local minimum of $\mathrm{v}_{\varphi}$ for $r$ for both $a_{l}$ and $a_{h}$ at the radii $r$ locating the resonance sites, thereby defining two different eigenstates. This effect has been named 'Aschenbach' effect by Stuchlik et al., 2005.

Coming back to the remark, that the analysis of the mass-frequency relations in terms of resonances involving Kerr angular momenta as well as orbital velocities might point to a gravity quantum effect, I refer to Niels Bohr's discovery of quantum theory, which involves angular momenta, especially orbital radii, natural numbers and the term $2 \pi$.

\section{References}

[1] B. Aschenbach, 2004, Measuring mass and angular momentum of black holes with high-frequency quasi-periodic oscillations, A\&A 425, 1075 - 1082.

[2] B.Aschenbach, 2018, A Model for the High and Low Frequency Oscillations in Black Hole Accretion Disks including Sgr $A^{*}$, in proceedings of the conference Frontier Research in Astrophysics - III (FRAPWS2018)28 May - 2 June 2018 Mondello (Palermo), Italy, POS (FRAPWS2018) 052.

[3] W. Becker, M. Kramer, A. Sesana, 2018, Pulsar Timing and its application for Navigation and Gravitational Wave Detection, Space Sci. Rev. 214:30. 
[4] J.W.T. Hessels, S.M. Ransom, I.H. Stairs, et al., 2006, A Radio Pulsar Spinning at $716 \mathrm{~Hz}$, Science 311, $1901-1904$.

[5] J.M. Lattimer \& M. Prakash, 2004, The Physics of Neutron Stars, Science 304, 536 -542 .

[6] K.L. Smith, R.F. Mushotzky, P.T. Boyd \& V.R. Wagoner 2018, Evidence for an Optical Low-frequency Quasi-periodic Oscillation in the Kepler Light Curve of an Active Galaxy, ApJ 860, L10 - L15.

[7] Z. Stuchlík, P. Slańy, G. Török, M.A. Abramowicz, 2005, Aschenbach effect: Unexpected topology changes in the motion of particles and fluids orbiting rapidly rotating Kerr black holes, Phys. Rev. 71, Issue 2, id. 024037. 